\title{
Análisis multitemporal y estadístico de parámetros fisicoquímicos monitoreados en la cuenca alta del río Bogotá
}

\section{A Multitemporal and statistical analysis of physicochemical parameters monitored in the upper basin of the Bogotá River}

\author{
Yuri Lilian Santamaría-Zarate ${ }^{1}$, Ivonne Angulo-de Castro ${ }^{2 a}$, Martha Melizza \\ Ordoñez-Díaz ${ }^{2 b}$
}

\begin{abstract}
${ }^{1}$ Especialización en Evaluación Ambiental de Proyectos, Universidad Manuela Beltrán, Colombia. Correo electrónico: yurisantamaria.1@ academia.umb.edu.co. Orcid: 0000-0003-0377-3896.

${ }^{2}$ Ingeniería Ambiental, Universidad Manuela Beltrán Colombia. Correo electrónico: a ivonne.angulo@umb.edu.co, b martha.ordonez@umb.edu.co. Orcid: ${ }^{\mathrm{a}}$ 0000-0002-3873-9674, ${ }^{\mathrm{b}}$ 0000-0002-3349-3070.
\end{abstract}

Recibido: 28 agosto, 2020. Aceptado: 12 marzo, 2021. Versión final: 12 junio, 2021.

\section{Resumen}

Las actividades antrópicas han dejado su huella de manera progresiva en el entorno del cual el hombre obtiene provecho de los bienes y servicios ecosistémicos, lo que ha trascendido a conflictos socioambientales a lo largo de la historia. Prueba de ello son los cambios drásticos sufridos por el río Bogotá a causa de los vertimientos sin control, provenientes del continuo crecimiento de las poblaciones y actividades aledañas al cuerpo de agua. En el presente estudio se expone la variabilidad de los parámetros ambientales en la cuenca alta del río Bogotá entre los años 2007 al 2019 luego de la expedición del Acuerdo 43 del 2006 de la CAR; se llevó a cabo un análisis multitemporal y estadístico de los datos. Los resultados evidencian que a lo largo de los doce años de monitoreo no se presenta contaminación a causa de nitratos, nitritos ni sulfatos en la cuenca alta del río Bogotá; de manera similar, el pH monitoreado se mantuvo dentro de los objetivos de calidad, a excepción de 3 años en los que se superó el valor máximo permitido en la parte superior de la cuenca. No obstante, el deterioro creciente de la calidad del agua en el río Bogotá se atribuye principalmente a los sólidos suspendidos totales que muestran un incumplimiento permanente en la cuenca alta superior e inferior, sumado a los incumplimientos en la cuenca alta inferior de los objetivos de calidad para los parámetros oxígeno disuelto, DBO y DQO. En consecuencia, los resultados evidencian el escaso impacto derivado de las acciones desarrolladas por parte de las autoridades locales y ambientales para asegurar los objetivos de calidad en la cuenca alta del río Bogotá, efecto de una débil gobernanza del agua en la cuenca.

Palabras clave: Acuerdo 43 de 2006; análisis multitemporal; calidad del agua; CAR; cuenca; estadística; límite; parámetros ambientales; río Bogotá; vertimientos.

\begin{abstract}
Anthropic activities have progressively left their mark in the environment where man benefits from the ecosystem goods and services, which has transcended socio-environmental conflicts throughout history. Proof of this is the drastic changes suffered by the Bogotá River due to the uncontrolled discharges from the continuous growth of populations and activities surrounding the body of water. In the present study, the variability of the environmental parameters in
\end{abstract}

ISSN impreso: 1657 - 4583. ISSN en línea: 2145 - 8456, CC BY-ND 4.0 @) (1) ()

Como citar: Y. L. Santamaría-Zarate, I. Angulo-de Castro, M. M. Ordoñez-Díaz, "Análisis multitemporal y estadístico de parámetros fisicoquímicos monitoreados en la cuenca alta del río Bogotá,” Rev. UIS Ing., vol. 20, no. 4, pp. 13-26, 2021, doi: 10.18273/revuin.v20n4-2021002. 
the upper basin of the Bogotá River between the years 2007 to 2019 is exposed after the issuance of the Agreement 43 of 2006 of the CAR, for which a multi-temporal analysis was carried out and statistical data. The results show that throughout the twelve (12) years of monitoring there is no contamination due to nitrates, nitrites, or sulfates in the upper basin of the Bogotá River, similarly, the monitored $\mathrm{pH}$ remained within the objectives of quality except for 3 years in which the maximum value allowed in the upper part of the basin was exceeded. However, the increasing deterioration of the water quality in the Bogotá River is mainly attributed to the total suspended solids that shows a permanent non-compliance in the upper and lower upper basin, added to the non-compliance in the lower upper basin according to the quality objectives for the dissolved oxygen, BOD and COD parameters. Consequently, the results show the scarce impact derived from the actions developed by the local and environmental authorities to ensure the quality objectives in the upper basin of the Bogotá River, and the effect of weak water governance in the basin.

Keywords: Agreement 43 of 2006; basin; Bogotá River; CAR; environmental parameters; flows; limit; multitemporal analysis; statistical; water quality.

\section{Introducción}

Las aguas naturales tienden a mantenerse en condiciones físicas, químicas y microbiológicas estables para la vida acuática y para el desarrollo de procesos biológicos y reacciones químicas. Sin embargo, con el paso del tiempo el ser humano ha dado usos diferentes al recurso hídrico, desde su captación para consumo hasta su inclusión en las actividades diarias, recreativas, productivas, de transporte y disposición de residuos. Lo anterior ha generado alteración en la calidad de las fuentes hídricas, reducción de la biodiversidad y una menor disponibilidad de agua limpia en el planeta como consecuencia de una actividad antrópica sin control.

Un claro ejemplo de ello es el río Bogotá en Colombia; una fuente hídrica ubicada en el altiplano cundiboyacense que nace en el páramo de Guacheneque en el municipio de Villapinzón y desemboca en el río Magdalena a la altura del municipio de Girardot; este río a finales de los años treinta aún "producía cangrejos suficientes para satisfacer el gusto gastronómico de todos los pueblos turísticos de la sabana y sus alrededores" [1].

Sus problemas de contaminación iniciaron hace ya más de 60 años, debido al crecimiento poblacional que se ha desarrollado en sus laderas tanto en el área rural como urbana, lo cual ha alterado su calidad y composición fisicoquímica, y ha generado cambios en su funcionalidad natural, producto de una cobertura incompleta del alcantarillado público; de la ausencia del tratamiento de las aguas residuales; del deterioro progresivo de los suelos por la sobreexplotación; del vertido de basuras, desechos agrícolas e industriales, y de la ausencia de medidas de protección y control ambiental para su conservación.

Aunque la recuperación del río Bogotá es estudiada desde el año 1974 [2] por parte del Ministerio de Medio Ambiente y Desarrollo Sostenible (MADS), la Corporación Autónoma Regional de Cundinamarca
(CAR), la Empresa de Acueducto y Alcantarillado de Bogotá e instituciones privadas y públicas de investigación, en la actualidad sus niveles de contaminación siguen siendo elevados; no obstante, en el municipio de Tocancipá aún se realiza captación de parte de sus aguas, siempre y cuando sus condiciones sean las adecuadas, para la potabilización y posterior suministro a la ciudad de Bogotá y a algunos municipios del departamento de Cundinamarca.

El $80 \%$ de la contaminación del río Bogotá está relacionada con los vertimientos industriales que realizan las curtiembres del municipio de Villapinzón en el departamento de Cundinamarca y por las aguas generadas en la ciudad de Bogotá; el $20 \%$ restante proviene de las industrias, la minería extractiva y los escombros [3].

La CAR [4] establece que, con el aumento de la demanda hídrica, se incrementan las descargas de aguas residuales domésticas e industriales, las cuales pueden estar asociadas con la calidad del agua en la parte alta de la cuenca. Frente a esto, la Secretaría Distrital de Planeación de Bogotá [5] resalta que uno de los aspectos más significativos relacionados con la degradación del río son las curtiembres que vierten una importante carga de materia orgánica a la fuente hídrica, lo cual va agotando el oxígeno disuelto del agua. En relación con esto, Rodríguez, González y Suárez [6] mencionan que las curtiembres en esta parte de la cuenca datan de 1820 $\mathrm{y}$ es una industria que con el paso del tiempo fue creciendo, especialmente en los municipios de Villapinzón y Chocontá. Por otro lado, en su estudio determinan que las aguas en la cuenca alta del río están contaminadas con metales que superan los límites que establece la Agencia de Protección Ambiental de los Estado Unidos, y que afectan y contaminan la fauna acuática que aún queda en el río. 
Sanabria, Montenegro, Castro y Díaz [7] mencionan que el uso excesivo de agroquímicos en la cuenca alta del río Bogotá también contribuye en el deterioro de la calidad de sus aguas.

En relación con la problemática ambiental que presenta el río Bogotá, en esta investigación se evalúa el comportamiento que mostraron los parámetros fisicoquímicos de calidad ambiental monitoreados (oxígeno disuelto, $\mathrm{DBO}_{5}$, sólidos suspendidos totales, nitratos, nitritos, $\mathrm{pH}$, sulfatos y plomo) y la clasificación de usos del agua en la parte alta de la cuenca durante los años 2007 al 2019, luego de que la CAR estableciera [8], a través en el Acuerdo n. ${ }^{\circ} 43$ del 17 de octubre de 2006, los objetivos de calidad del agua para la cuenca alta, media y baja del río Bogotá a lograr en el año 2020.

En este sentido, se plantea un análisis multitemporal y estadístico de los datos de diferentes estudios realizados al río Bogotá durante los últimos 12 años, cuyo método "permite detectar cambios entre diferentes fechas de referencia, deduciendo la evolución del medio natural o las repercusiones de la acción humana sobre ese medio" [9]. Es importante mencionar que los datos del análisis multitemporal, "varían en el número de fechas de análisis empleadas (más de dos), y se emplean técnicas estadísticas de series de tiempo para su análisis" [10], por lo cual, para el objeto del presente estudio, es la técnica más adecuada para el análisis de la información.

Lo anterior se lleva a cabo a través de la consulta, recolección, organización y selección de datos; posterior, se representan de manera gráfica y se comparan normativamente; luego, se analizan de manera cuantitativa, estadística y ordinal cualitativa, y, finalmente, se obtienen los resultados y conclusiones, que harán posible llegar a la respuesta del interrogante: ¿han sido eficaces las acciones establecidas por las autoridades ambientales para el cumplimiento de los objetivos planteados para la cuenca alta del río Bogotá en el Acuerdo 43 de 2006 de la CAR?

\section{Metodología}

Se empleó un diseño no experimental con enfoque mixto para el análisis multitemporal y estadístico al año 2019 de las concentraciones fisicoquímicas obtenidas de diferentes estudios realizados de la calidad del agua en la cuenca alta del río Bogotá. Para este propósito se plantean las siguientes seis fases.

- Fase 1. Consulta y recolección de datos: se solicitaron los resultados de los análisis fisicoquímicos de los diferentes estudios realizados en la cuenca alta del río Bogotá entre el 2007 al año 2019 por entidades oficiales como la CAR y el MADS; se extrajo información también de la literatura disponible en bases de datos en línea y otras fuentes de información secundaria confiable encontrada en internet.

- Fase 2. Selección de la información: la información recolectada en la fase anterior se organizó empleando una ficha RAE (resumen de análisis especializado), de acuerdo con los parámetros establecidos para medir la calidad de las aguas superficiales.

- Fase 3. Representación gráfica y comparación normativa: se efectuó la representación multitemporal en gráficas de Excel con la respectiva comparación normativa. En este caso, se estableció la comparación con los parámetros de calidad aplicables a la localización de los puntos de muestreo, que en su totalidad corresponden a los parámetros para los usos clase II.

- Fase 4. Análisis cuantitativo y estadística: con los datos depurados, ordenados según su ubicación en la cuenca alta superior o cuenca alta inferior, se realizó el cálculo de las variables (máximo, mínimo, promedio, porcentajes y desviación estándar de los datos). Se elaboro el diagrama cuantil-cuartil, para mayor facilidad en el manejo de la información, dividiendo los datos en dos grupos: a) parámetros físicos con restricción normativa, b) parámetros químicos con concentración máxima permitida.

- Fase 5. Análisis ordinal cualitativo: con un sentido de escala se sitúan los datos que presentan cada parámetro.

- Fase 6. Resultados y análisis: a partir de los resultados obtenidos del análisis cualitativo y cuantitativo, se da respuesta a la pregunta de investigación sobre si durante los once años estudiados en la presente investigación, la expedición del Acuerdo 43 del 2006 de la CAR logró que se tomaran medidas que contribuyan a la recuperación de la cuenca alta del río Bogotá.

\section{Resultados}

El MADS [11] prohíbe vertimientos sin previo tratamiento, con el fin de evitar la contaminación o la eutrofización de las aguas, causar daño a la salud humana u obstaculizar el empleo de la fuente hídrica en otros usos; toda actividad que genere vertimientos a las aguas superficiales deberá tener su respectivo permiso de vertimientos.

La Empresa de Acueducto y Alcantarillado de Bogotá y la Universidad Nacional [12] determinaron que en la parte superior de la cuenca alta del río Bogotá hasta la 
estación de Saucío la variación de la calidad del agua se debe a la influencia de los vertimientos de las curtiembres; en relación con esto, en el presente estudio se evalúa cuál fue el comportamiento posterior de los parámetros físicoquímicos y microbiológicos en esta parte de la cuenca.

Díaz y Granada [13] abordan el efecto de las actividades antrópicas sobre el río Bogotá a lo largo del municipio de Villapinzón; este estudio permitió establecer que en todas las estaciones evaluadas los resultados microbiológicos presentan concentraciones por encima del nivel saludable. Además, el contenido de cromo total en la zona de influencia de las curtiembres supera el límite máximo, sumado al deterioro progresivo de la calidad del agua asociado a la alta prevalencia de enfermedades infecciosas y parasitarias del sistema digestivo en niños mayores a cinco años del municipio.

Por otro lado, en el informe técnico de la implementación de estrategias de producción más limpia en la cuenca alta del río Bogotá, la Universidad de la Salle [14] señala que con los monitoreos de la calidad de las aguas lo más común fue la presencia de metales pesados; también identificaron las concentraciones de detergentes y fenoles.
El río Bogotá recorre 46 municipios del departamento de Cundinamarca y específicamente en su cuenca alta, atraviesa los municipios de Villapinzón, Chocontá, Suesca, Sesquilé, Gachancipá, Tocancipá, Cajicá, Chía y Cota, en cuyo recorrido se encuentran 24 estaciones de monitoreo, las cuales son el principal instrumento de recolección de información para el presente estudio (véase figura 1).

Es importante mencionar que los parámetros fisicoquímicos del agua, extraídos de las 24 estaciones de monitoreo, para el presente estudio son: concentraciones de plomo, oxígeno, $\mathrm{DBO}_{5}$, sólidos suspendidos totales, nitratos, nitritos, $\mathrm{pH}$ y sulfatos entre los años 2007 al 2019, y las referenciadas en el Acuerdo n. ${ }^{\circ} 43$ del 2006 de la CAR, tomadas como línea base y comparadas con los objetivos de calidad a cumplir al año 2020 para el tramo I.

En la figura 2 se evidencia que el elemento metálico plomo en el año 2006 tiene una incidencia representativa en el tramo Tibitoc - Estación hidrometeorológica la Virgen, al sobrepasar considerablemente los 0,05 mg/L, expuesto como meta proyectada para esta parte de la cuenca del río Bogotá.

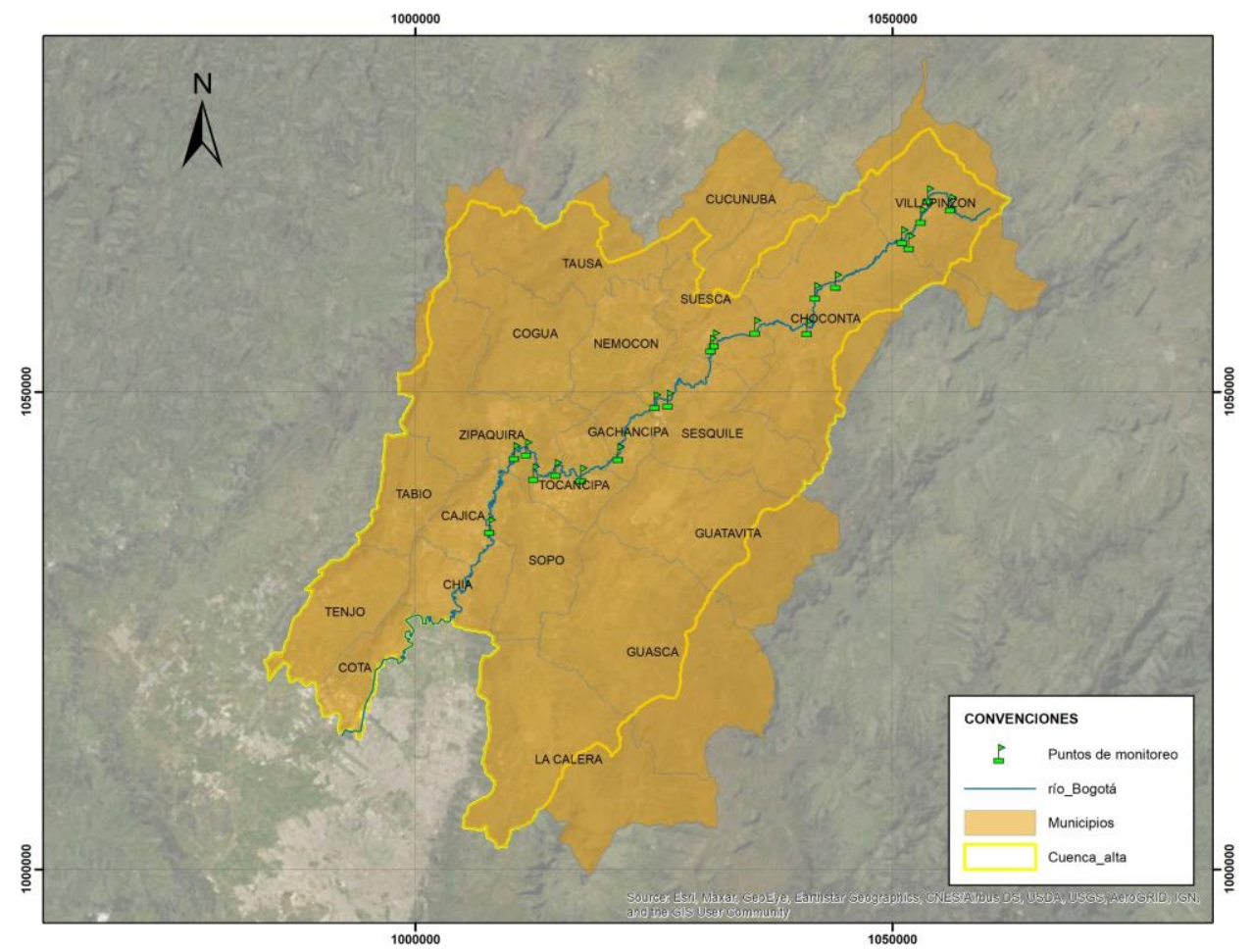

Figura 1. Puntos monitoreados cuenca alta río Bogotá. Fuente: elaboración propia. 
Análisis multitemporal y estadístico de parámetros fisicoquímicos monitoreados en la cuenca alta del río Bogotá

Tabla 1. Concentraciones de $\mathrm{O}_{2}$ en $\mathrm{mg} \mathrm{O}_{2} / \mathrm{L}$ en la cuenca alta, año 2006-2017

\begin{tabular}{|c|c|c|c|c|c|c|c|c|c|c|}
\hline Cuenca alta & \multicolumn{9}{|c|}{ Superior } & \multicolumn{4}{c|}{ Inferior } \\
\hline Año & Vr. Mín. & Vr. Máx. & Promedio & S & Cumplimiento & Vr. Mín. & Vr. Máx. & Promedio & S & Cumplimiento \\
\hline $\mathbf{2 0 0 6}$ & 6 & 6 & 6 & 0 & - & 2 & 6 & 4 & 2,83 & - \\
\hline $\mathbf{2 0 0 7}$ & 4,1 & 9,8 & 6,69 & 1,8 & $100 \%$ & 0,7 & 5,6 & 3,09 & 1,6 & $37,5 \%$ \\
\hline $\mathbf{2 0 0 8}$ & 1,25 & 7,8 & 5,36 & 1,6 & $90,6 \%$ & 0,8 & 4,7 & 2,27 & 1,3 & $8,3 \%$ \\
\hline $\mathbf{2 0 0 9}$ & 2,7 & 15,9 & 7,82 & 3,5 & $92,0 \%$ & 1 & 3,7 & 1,91 & 1,1 & $0,0 \%$ \\
\hline $\mathbf{2 0 1 0}$ & 2,4 & 7,8 & 5,26 & 1,6 & $80,6 \%$ & 0,5 & 5,2 & 2,11 & 1,8 & $27,3 \%$ \\
\hline $\mathbf{2 0 1 1}$ & 2,7 & 7,6 & 5,75 & 1,2 & $96,9 \%$ & 0 & 4,5 & 1,82 & 1,4 & $10,0 \%$ \\
\hline $\mathbf{2 0 1 2}$ & 1,8 & 8,1 & 5,92 & 1,3 & $93,1 \%$ & 0,5 & 4,5 & 1,97 & 1,5 & $23,5 \%$ \\
\hline $\mathbf{2 0 1 3}$ & 0,6 & 8,3 & 6,15 & 1,4 & $93,3 \%$ & 0,5 & 5,2 & 1,93 & 1,6 & $23,5 \%$ \\
\hline $\mathbf{2 0 1 4}$ & 2 & 7,5 & 5,24 & 1,2 & $84,2 \%$ & 0,7 & 5,9 & 2,35 & 1,8 & $27,3 \%$ \\
\hline $\mathbf{2 0 1 5} *$ & 2 & 6,9 & 5,49 & 1,7 & $88,9 \%$ & 1,3 & 6,8 & 3,7 & 2,4 & $50,0 \%$ \\
\hline $\mathbf{2 0 1 6}$ & 4,2 & 7,5 & 5,68 & 1,8 & $88,9 \%$ & 1 & 5,6 & 3,62 & 1,8 & $50,0 \%$ \\
\hline $\mathbf{2 0 1 7} *$ & 2,2 & 7,5 & 6,87 & 1,1 & $100,0 \%$ & 1,2 & 5,2 & 4,02 & 1,9 & $75,0 \%$ \\
\hline
\end{tabular}

S: desviación estándar. -: dato no determinado. *Se contemplaron datos al menos de cuatro (4) estaciones, para los demás años se contemplaron al menos veintidós (22) estaciones.

Fuente: elaboración propia.

Rodríguez, González y Suárez [6] en su estudio confirman que muchos de los metales encontrados en el agua del río Bogotá provienen de las curtiembres que vierten sus efluentes a este cuerpo de agua.

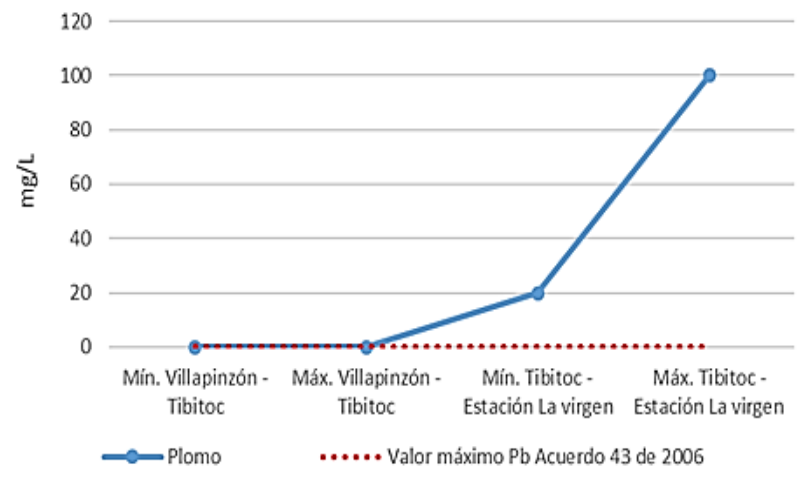

Figura 2. Concentración plomo año 2006 vs. norma. Fuente: elaboración propia.

En los estudios realizados entre los años 2010 al 2017, las mediciones reportaron valores menores a los límites de cuantificación de la técnica empleada para su análisis, con lo que se establece que se ha dejado de verter estas aguas residuales o que se han tratado para reducir al máximo su concentración.

En relación con los datos analizados, en la tabla 1 es posible observar que las concentraciones mínimas registradas de oxígeno disuelto en la cuenca alta estuvieron por debajo de la cifra referenciada en el Acuerdo 43/06; en el caso de los valores máximos, su comportamiento se mantuvo, al reportar contenidos superiores a $6 \mathrm{mg} / \mathrm{L}$ en la parte superior de la cuenca, lo cual favorece el desarrollo de la fauna acuática.
En este mismo tramo, para el año 2009, se evidencia el mayor distanciamiento de los datos individuales en comparación con el valor promedio, mientras que en la parte inferior de la cuenca este comportamiento se registra en el año 2006 y se vuelve a presentar de manera similar para el año 2015.

De acuerdo con los datos analizados para cada uno de los años, es posible observar en la tabla 1 un importante cumplimiento de la concentración de $\mathrm{O}_{2}$ en la parte alta superior de la cuenca, mientras que en la parte inferior las concentraciones de oxígeno disuelto siguen siendo inferiores a los objetivos de calidad, lo cual afecta el hábitat para las diferentes especies de organismos aerobios que dependen del oxígeno para sus procesos biológicos. En la figura 3 se plasma el consolidado de las concentraciones de $\mathrm{O}_{2}$ de acuerdo con los datos analizados para los años 2007 al 2017.

En el caso de las concentraciones estudiadas de $\mathrm{DBO}_{5}$, en la tabla 2 se establece que los valores mínimos estuvieron por debajo de los datos de referencia del 2006 y del límite máximo de $10 \mathrm{mg} \mathrm{O}_{2} / \mathrm{L}$ establecido por Acuerdo 43/06. En el caso de los registros más altos, se mantuvieron por encima del objetivo de calidad hasta el año 2013; en los años 2014, 2016 y 2017 en la cuenca alta inferior se observó cumplimiento, mientras que en la cuenca alta superior el contenido máximo adecuado se dio solo en 2016, lo cual representa los altos requerimientos de oxígeno disuelto de los microorganismos presentes en el agua para realizar la oxidación biológica de la materia orgánica contenida en el cuerpo hídrico. 


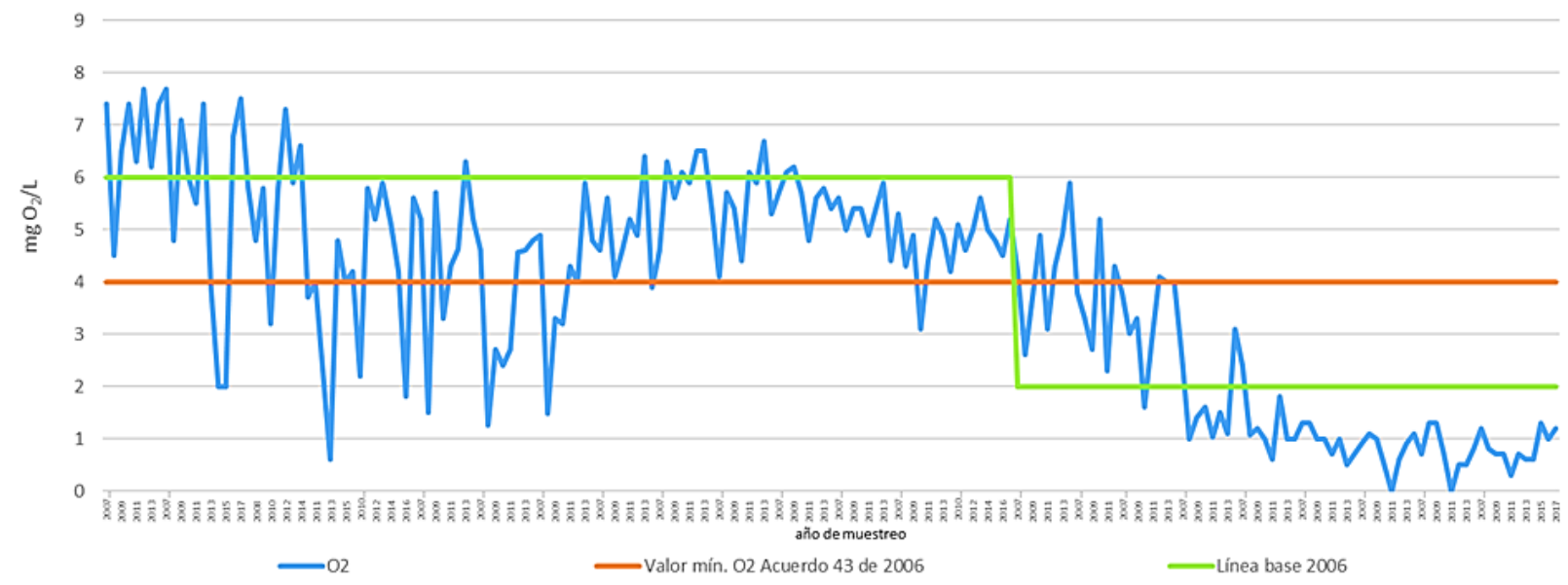

Figura 3. Comportamiento oxígeno disuelto desde 2007 al 2017 vs. norma. Fuente: elaboración propia.

Tabla 2. Concentraciones de $\mathrm{DBO}_{5}$ en $\mathrm{mg} \mathrm{O} / \mathrm{L}$ cuenca alta año 2006-2017

\begin{tabular}{|c|c|c|c|c|c|c|c|c|c|c|}
\hline $\begin{array}{c}\text { Cuenca } \\
\text { alta }\end{array}$ & \multicolumn{5}{|c|}{ Superior } & \multicolumn{5}{|c|}{ Inferior } \\
\hline Año & Vr. Mín. & Vr. Máx. & Promedio & $\mathbf{S}$ & Cumplimiento & Vr. Mín. & Vr. Máx. & Promedio & $\mathbf{S}$ & Cumplimiento \\
\hline 2006 & 20 & 70 & 45 & 35,36 & - & 70 & 150 & 110 & 56,57 & - \\
\hline 2007 & 2 & 43,4 & 8,55 & 8,77 & $76,9 \%$ & 2,4 & 19 & 7,46 & 4,58 & $75,0 \%$ \\
\hline 2008 & 1,7 & 25 & 5,22 & 5,36 & $90,6 \%$ & 3,3 & 17 & 7,89 & 3,72 & $83,3 \%$ \\
\hline 2009 & 2 & 11,6 & 4,17 & 3,56 & $84,6 \%$ & 2,2 & 34,7 & 11,64 & 10,18 & $62,5 \%$ \\
\hline 2010 & 2 & 99,3 & 10,53 & 20,65 & $84,4 \%$ & 2 & 243 & 24,79 & 63,51 & $64,3 \%$ \\
\hline 2011 & 2 & 52,2 & 6,14 & 9,60 & $86,7 \%$ & 2,4 & 33,8 & 7,86 & 6,95 & $75,0 \%$ \\
\hline 2012 & 2 & 22 & 5,74 & 5,22 & $85,2 \%$ & 2 & 19,5 & 8,17 & 5,18 & $58,8 \%$ \\
\hline 2013 & 2 & 14,2 & 4,78 & 3,62 & $83,3 \%$ & 2 & 18 & 7,5 & 4,97 & $77,8 \%$ \\
\hline 2014 & 2 & 10 & 4,02 & 2,22 & $100,0 \%$ & 2,5 & 50,7 & 13,46 & 14,67 & $60,0 \%$ \\
\hline 2015* & 2 & 39 & 13,5 & 17,14 & $75,0 \%$ & 2 & 16 & 8,5 & 6,12 & $66,7 \%$ \\
\hline 2016* & 4,8 & 7,5 & 6,58 & 1,22 & $100,0 \%$ & 4,8 & 8,9 & 7,1 & 2,55 & $100,0 \%$ \\
\hline 2017* & 5,6 & 7,5 & 6,77 & 1,02 & $100,0 \%$ & 3 & 19 & 11 & 11,31 & $50,0 \%$ \\
\hline
\end{tabular}

S: desviación estándar. -: dato no determinado. * Se contemplaron datos al menos de cuatro (4) estaciones. Para los demás años se contemplaron al menos veintidós (22) estaciones.

Fuente: elaboración propia.

En este último tramo mencionado, el distanciamiento más representativo de los datos individuales con relación al valor promedio se registra en el año 2006, seguido del año 2010 y 2015; en cambio en la parte inferior de la cuenca este comportamiento estuvo más marcado en el año 2010, seguido por el año 2006.

De acuerdo con los datos analizados en la tabla 2, se observa que a partir del año 2008 la $\mathrm{DBO}_{5}$ indicó un cumplimiento representativo en la parte alta superior de la cuenca, mientras que en la parte inferior las concentraciones siguen siendo superiores al objetivo de calidad. Este comportamiento se observa principalmente en las estaciones Aguas Abajo Río Negro, cuyo afluente presenta una carga contaminante generada por el municipio de Zipaquirá [15]; y desde Aguas Abajo Chía, lo cual puede estar asociado al vertimiento de la PTAR municipal de Chía [16] hasta el puente la Virgen, en donde existen descargas industriales y domésticas [12].

En la figura 4 se plasma el consolidado de las concentraciones de $\mathrm{DBO}_{5}$, de acuerdo con los datos analizados para los años 2007 al 2017.

Por otra parte, en relación con los datos analizados, es posible establecer de acuerdo con la tabla 3 que las concentraciones mínimas registradas de sólidos suspendidos totales en la cuenca alta superior estuvieron por debajo de la cifra referenciada para el año 2006 y del límite máximo de $10 \mathrm{mg} / \mathrm{L}$ del Acuerdo 43/06; en el caso de los valores máximos, su comportamiento se mantuvo por encima del objetivo de calidad. 


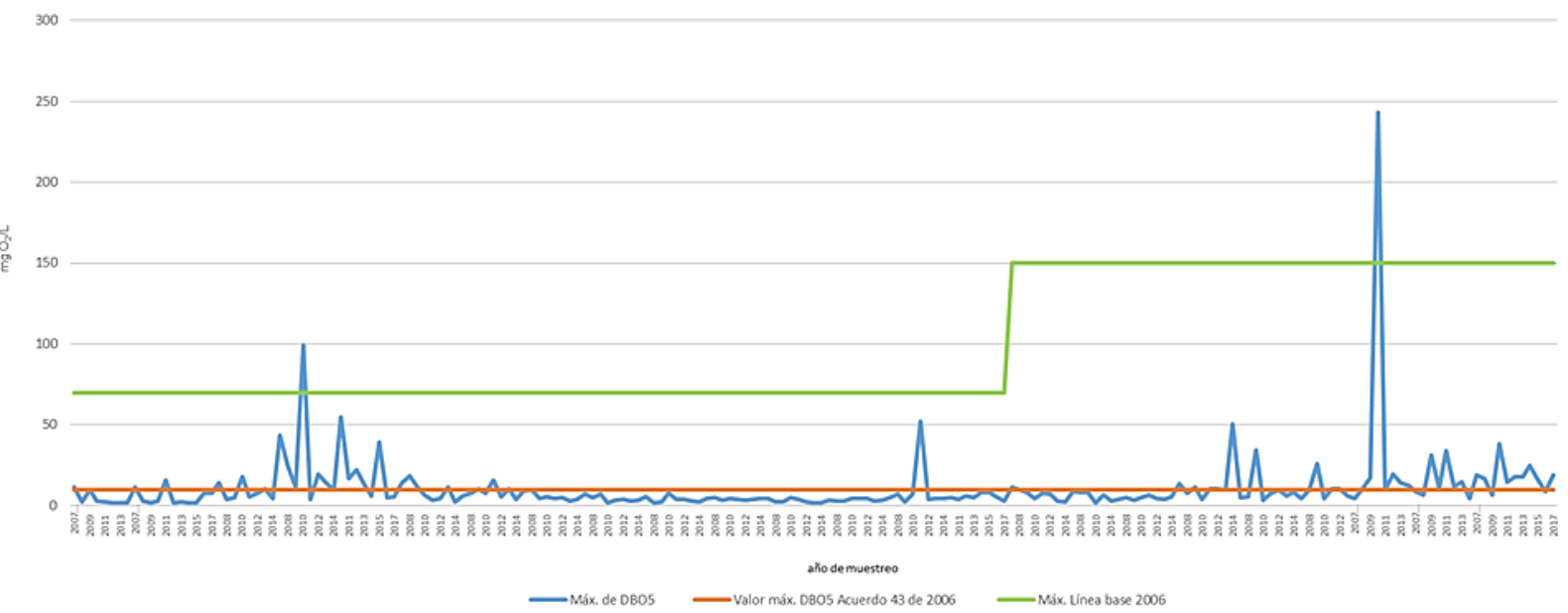

Figura 4. Comportamiento $\mathrm{DBO}_{5}$ desde 2007 al 2017 vs. norma. Fuente: elaboración propia.

Tabla 3. Concentraciones de SST en mg/L cuenca alta año 2006-2017

\begin{tabular}{|c|c|c|c|c|c|c|c|c|c|c|}
\hline $\begin{array}{c}\text { Cuenca } \\
\text { alta }\end{array}$ & \multicolumn{9}{|c|}{ Superior } & \multicolumn{5}{c|}{ Inferior } \\
\hline Año & Vr. Mín. & Vr. Máx. & Promedio & S & Cumplimiento & Vr. Mín. & Vr. Máx. & Promedio & S & Cumplimiento \\
\hline $\mathbf{2 0 0 6}$ & 20 & 100 & 60 & 56,57 & - & 20 & 100 & 60 & 56,57 & - \\
\hline $\mathbf{2 0 0 7}$ & 5 & 72,7 & 33,92 & 19,34 & $9,5 \%$ & 15,2 & 65,9 & 31,13 & 14,15 & $0,0 \%$ \\
\hline $\mathbf{2 0 0 8}$ & 2,7 & 198 & 37,20 & 41,49 & $28,1 \%$ & 13,8 & 156 & 46,66 & 40,15 & $0,0 \%$ \\
\hline $\mathbf{2 0 0 9}$ & 5 & 47,3 & 23,96 & 12,95 & $15,4 \%$ & 13 & 160 & 53,99 & 47,56 & $0,0 \%$ \\
\hline $\mathbf{2 0 1 0}$ & 4,5 & 188 & 44,72 & 50,41 & $25,8 \%$ & 5 & 281 & 48,93 & 73,64 & $7,7 \%$ \\
\hline $\mathbf{2 0 1 1}$ & 0 & 160 & 43,02 & 32,79 & $15,6 \%$ & 18,6 & 290 & 76,15 & 76,66 & $0,0 \%$ \\
\hline $\mathbf{2 0 1 2}$ & 4,5 & 430 & 110,47 & 126,48 & $10,3 \%$ & 29 & 111 & 56,03 & 22,93 & $0,0 \%$ \\
\hline $\mathbf{2 0 1 3}$ & 4 & 69,1 & 17,03 & 13,23 & $31,3 \%$ & 12,4 & 72 & 36,22 & 16,83 & $0,0 \%$ \\
\hline $\mathbf{2 0 1 4}$ & 4,5 & 47 & 23,43 & 13,13 & $16,7 \%$ & 14 & 48,6 & 24,04 & 11,35 & $0,0 \%$ \\
\hline $\mathbf{2 0 1 5} *$ & 4,5 & 23 & 11,53 & 6,92 & $50,0 \%$ & 17 & 140 & 45,5 & 47,62 & $0,0 \%$ \\
\hline $\mathbf{2 0 1 6} *$ & 4,5 & 27 & 9,18 & 6,20 & $66,7 \%$ & 18 & 65 & 40,83 & 20,05 & $0,0 \%$ \\
\hline $\mathbf{2 0 1 7}$ & 4,5 & 40 & 10 & 9,53 & $66,7 \%$ & 22 & 41 & 35,5 & 9,04 & $0,0 \%$ \\
\hline
\end{tabular}

S: desviación estándar. -: dato no determinado. * Se contemplaron datos al menos de cuatro (4) estaciones.

Para los demás años se contemplaron al menos veintidós (22) estaciones.

Fuente: elaboración propia.

En la cuenca alta superior el distanciamiento de los datos individuales en comparación con el valor promedio fue más representativo, en este mismo orden, para los años 2012, 2006, 2010, 2008 y 2011; mientras que en la parte inferior de la cuenca alta este comportamiento estuvo más marcado en los años 2011 y 2010, seguido del 2006.

De acuerdo con los datos analizados, en la tabla 3 se observa que predomina el incumplimiento del objetivo de calidad ( $<10 \mathrm{mg} / \mathrm{L}$ ) para los sólidos suspendidos totales; este comportamiento está más marcado en la parte inferior de la cuenca alta, en donde el único año que evidenció un leve cumplimiento fue el año 2010. En relación con lo establecido por Suárez, García y Vaca [17], el aporte de sólidos se debe a los vertimientos, principalmente de la industria de curtiembres, sin embargo, también está estrechamente relacionado con los periodos de lluvia en la cuenca de la fuente monitoreada.

En la figura 5 se plasma el consolidado de los resultados para cada una de las mediciones, de acuerdo con los datos analizados para los años 2007 al 2017.

En relación con los nutrientes, es posible observar en la tabla 4 y tabla 5 que las concentraciones de nitratos y nitritos no han sido representativas en cada uno de los años estudiados, y que el $100 \%$ de estas han estado por debajo del límite máximo de $10 \mathrm{mg} / \mathrm{L}$, fijado para cada uno de estos parámetros en el Acuerdo 43/06. 


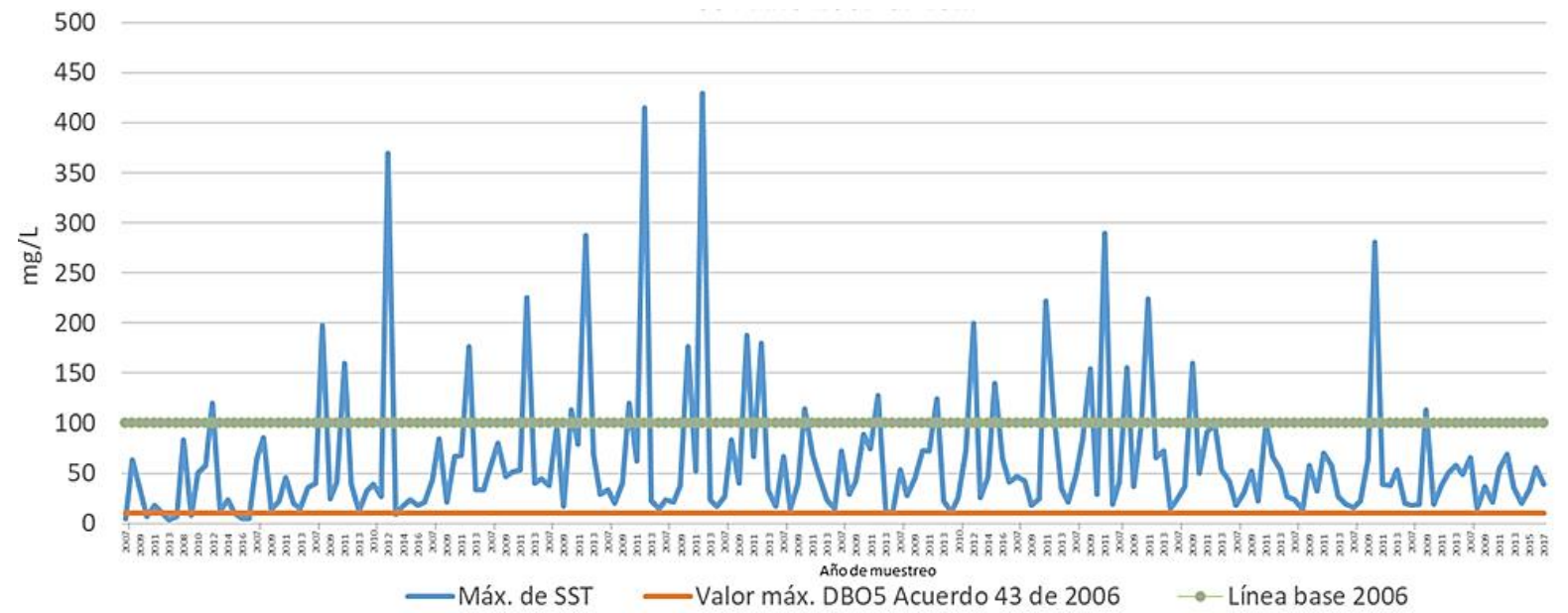

Figura 5. Comportamiento SST desde 2007 al 2017 vs. norma. Fuente: elaboración propia.

Tabla 4. Concentraciones de nitratos en $\mathrm{mg} \mathrm{NO}$ - $\mathrm{N} / \mathrm{L}$, cuenca alta, año 2007-2019

\begin{tabular}{|c|c|c|c|c|c|c|c|c|}
\hline Cuenca alta & \multicolumn{4}{|c|}{ Superior } & \multicolumn{4}{c|}{ Inferior } \\
\hline Año & Vr. Mín. & Vr. Máx. & Promedio & S & Vr. Mín. & Vr. Máx. & Promedio & S \\
\hline $\mathbf{2 0 0 7}$ & 0 & 1,4 & 0,44 & 0,43 & 0 & 1 & 0,52 & 0,30 \\
\hline $\mathbf{2 0 0 8}$ & 0,025 & 1,3 & 0,52 & 0,35 & 0,3 & 0,8 & 0,49 & 0,17 \\
\hline $\mathbf{2 0 0 9}$ & 0,1 & 1 & 0,49 & 0,28 & 0,2 & 0,9 & 0,58 & 0,24 \\
\hline $\mathbf{2 0 1 0}$ & 0,1 & 2,3 & 0,84 & 0,62 & 0,1 & 1,3 & 0,46 & 0,36 \\
\hline $\mathbf{2 0 1 1}$ & 0,1 & 2,2 & 0,50 & 0,39 & 0,1 & 1,4 & 0,65 & 0,36 \\
\hline $\mathbf{2 0 1 2}$ & 0,05 & 1,7 & 0,62 & 0,41 & 0,1 & 0,9 & 0,53 & 0,23 \\
\hline $\mathbf{2 0 1 3}$ & 0,1 & 0,9 & 0,51 & 0,26 & 0,2 & 1,1 & 0,61 & 0,26 \\
\hline $\mathbf{2 0 1 4}$ & 0,1 & 1,6 & 0,64 & 0,51 & 0,2 & 1,23 & 0,67 & 0,39 \\
\hline $\mathbf{2 0 1 5}$ & 0,07 & 0,7 & 0,24 & 0,16 & 0,15 & 0,6 & 0,43 & 0,21 \\
\hline $\mathbf{2 0 1 6}$ & 0,075 & 0,82 & 0,35 & 0,27 & 0,29 & 0,73 & 0,59 & 0,27 \\
\hline $\mathbf{2 0 1 7}$ & 0,19 & 0,98 & 0,30 & 0,17 & 0,17 & 1 & 0,63 & 0,42 \\
\hline $\mathbf{2 0 1 9}$ & - & 0,4 & N/A & N/A & - & - & N/A & N/A \\
\hline
\end{tabular}

S: desviación estándar; -:sin registro; N/A: no aplica.

Fuente: elaboración propia.

Tabla 5. Concentraciones de nitritos en $\mathrm{mg} \mathrm{NO}$ - $\mathrm{N} / \mathrm{L}$, cuenca alta, año 2007-2019

\begin{tabular}{|c|c|c|c|c|c|c|c|c|}
\hline Cuenca alta & \multicolumn{4}{|c|}{ Superior } & \multicolumn{4}{c|}{ Inferior } \\
\hline Año & Vr. Mín. & Vr. Máx. & Promedio & S & Vr. Mín. & Vr. Máx. & Promedio & S \\
\hline $\mathbf{2 0 0 7}$ & 0,001 & 0,035 & 0,014 & 0,43 & 0 & 0,071 & 0,033 & 0,30 \\
\hline $\mathbf{2 0 0 8}$ & 0,001 & 0,049 & 0,015 & 0,35 & 0,014 & 0,047 & 0,030 & 0,17 \\
\hline $\mathbf{2 0 0 9}$ & 0,004 & 0,093 & 0,020 & 0,28 & 0,004 & 0,071 & 0,034 & 0,24 \\
\hline $\mathbf{2 0 1 0}$ & 0,004 & 0,049 & 0,016 & 0,62 & 0,004 & 0,046 & 0,020 & 0,36 \\
\hline $\mathbf{2 0 1 1}$ & 0,004 & 0,069 & 0,018 & 0,39 & 0,004 & 0,063 & 0,037 & 0,36 \\
\hline $\mathbf{2 0 1 2}$ & 0,004 & 0,054 & 0,019 & 0,41 & 0,021 & 0,093 & 0,062 & 0,23 \\
\hline $\mathbf{2 0 1 3}$ & 0,004 & 0,062 & 0,019 & 0,26 & 0,004 & 0,131 & 0,045 & 0,26 \\
\hline $\mathbf{2 0 1 4}$ & 0,004 & 0,225 & 0,030 & 0,51 & 0,004 & 0,092 & 0,028 & 0,39 \\
\hline $\mathbf{2 0 1 5}$ & 0,006 & 0,014 & 0,009 & 0,16 & 0,006 & 0,016 & 0,009 & 0,21 \\
\hline $\mathbf{2 0 1 6}$ & 0,006 & 0,022 & 0,009 & 0,27 & 0,006 & 0,058 & 0,021 & 0,27 \\
\hline $\mathbf{2 0 1 7}$ & 0,006 & 0,018 & 0,007 & 0,17 & 0,01 & 0,062 & 0,030 & 0.42 \\
\hline $\mathbf{2 0 1 9}$ & - & 0,24 & N/A & N/A & - & - & N/A & N/A \\
\hline
\end{tabular}

S: desviación estándar; -: sin registro; N/A: no aplica.

Fuente: elaboración propia. 
En este sentido, es importante mencionar que las aguas de la cuenca alta del río Bogotá no se encuentran contaminadas con nitratos ni nitritos y, por el contrario, las concentraciones permitidas de estos nutrientes pueden favorecer el rendimiento de las plantaciones que se alimentan de las aguas del este río.

En las figuras 6 y 7 se plasma el consolidado de las concentraciones de nitratos y nitritos (respectivamente) de los datos analizados para los años 2007 al 2019.

De los datos estudiados de $\mathrm{pH}$, de acuerdo con la tabla 6, se establece que los valores mínimos en la cuenca alta estuvieron dentro de los objetivos de calidad para el año 2020, mientras que, en los años 2010, 2012 y 2016, los registros más altos en la parte superior de la cuenca se mantuvieron por encima del límite máximo de
9 unidades; dichas variaciones en el $\mathrm{pH}$, puede generar "cambios en la fauna y la flora de los cuerpos de agua, influencia sobre la toxicidad de ciertos compuestos, como el amoniaco, metales pesados, hidrógeno sulfurados, reacciones secundarias, rompiendo el ciclo ecológico en un cuerpo de agua" [18], lo anterior, a causa de su influencia en el equilibrio de la mayoría de compuestos químicos, entre otras características del agua [19].

En cuanto al mayor distanciamiento de los datos individuales, en comparación con el valor promedio, este se dio en los años 2010 y 2012 en la parte superior de la cuenca.

En la figura 8 se plasma el resultado de pH de los datos analizados para los años 2007 al 2017.

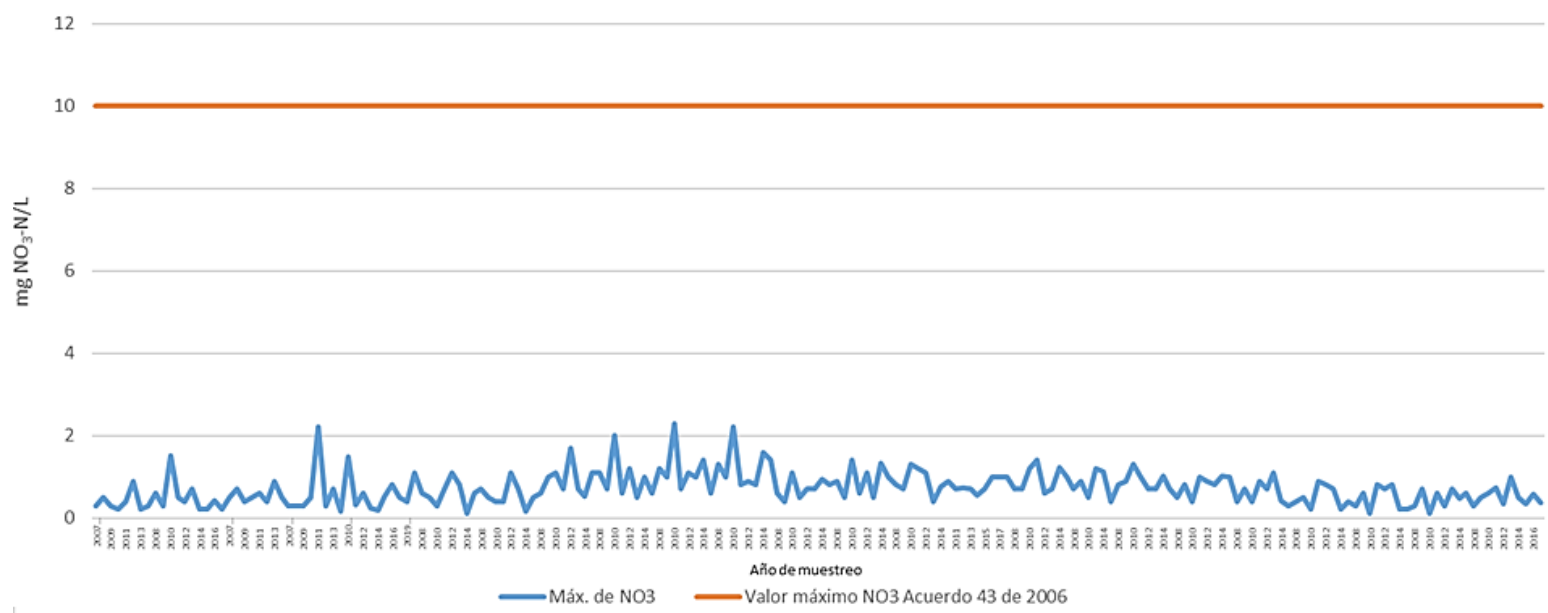

Figura 6. Comportamiento de nitratos desde 2007 al 2019 vs. norma. Fuente: elaboración propia.

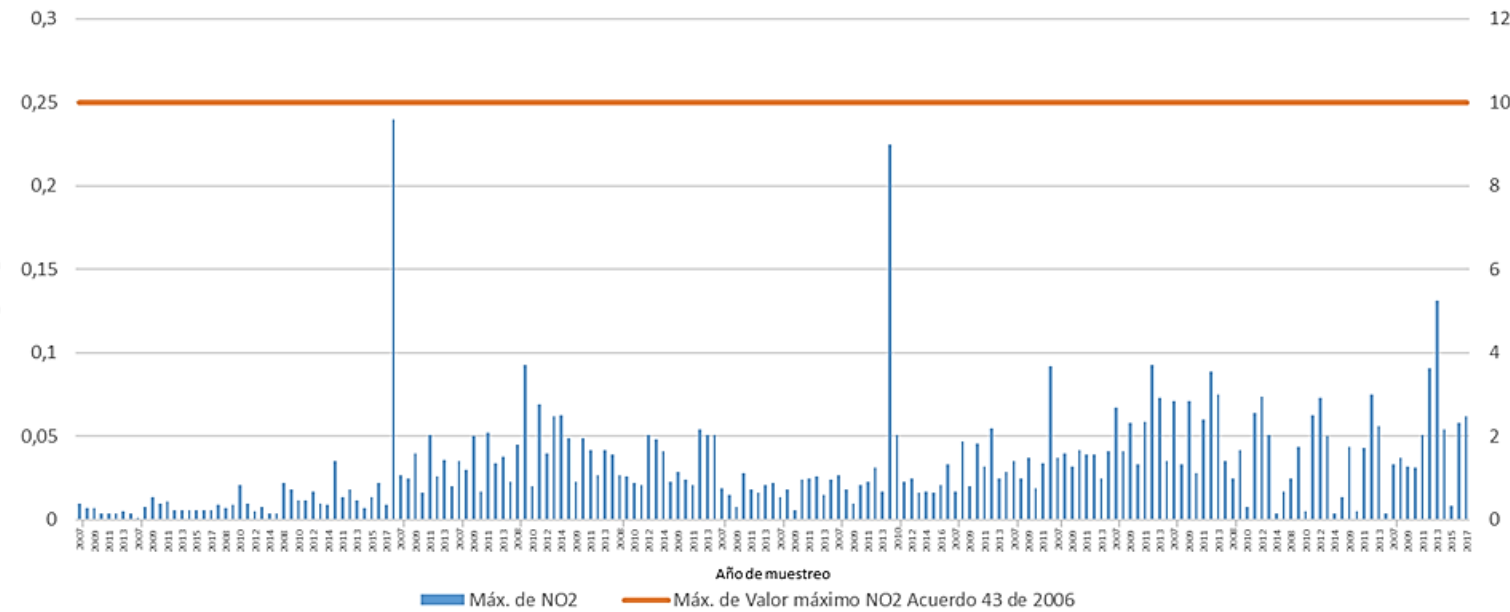

Figura 7. Comportamiento de nitritos desde 2007 al 2019 vs. norma. Fuente: elaboración propia. 
Tabla 6. Cuenca alta desde 2010 al 2017, pH en unidades

\begin{tabular}{|c|c|c|c|c|c|c|c|c|}
\hline Cuenca alta & \multicolumn{4}{|c|}{ Superior } & \multicolumn{4}{c|}{ Inferior } \\
\hline Año & Vr. Mín. & Vr. Máx. & S & Cumplimiento & Vr. Mín. & Vr. Máx. & S & Cumplimiento \\
\hline $\mathbf{2 0 1 0}$ & 6,88 & 9,62 & 1,28 & $75,0 \%$ & 6,39 & 7,13 & 0,38 & $100,0 \%$ \\
\hline $\mathbf{2 0 1 1}$ & 6,47 & 7,17 & 0,30 & $100,0 \%$ & 6,34 & 6,96 & 0,26 & $100,0 \%$ \\
\hline $\mathbf{2 0 1 2}$ & 5,95 & 9,22 & 1,56 & $75,0 \%$ & 6,18 & 7,11 & 0,40 & $100,0 \%$ \\
\hline $\mathbf{2 0 1 3}$ & 6,27 & 7,95 & 0,66 & $100,0 \%$ & 7,03 & 7,28 & 0,45 & $100,0 \%$ \\
\hline $\mathbf{2 0 1 4}$ & 6,54 & 7,29 & 0,32 & $100,0 \%$ & 6, & 7,16 & 0,17 & $100,0 \%$ \\
\hline $\mathbf{2 0 1 5}$ & 6,74 & 8,6 & 0,70 & $100,0 \%$ & 6,69 & 7,24 & 0,20 & $100,0 \%$ \\
\hline $\mathbf{2 0 1 6}$ & 6,63 & 9,01 & 0,84 & $75,0 \%$ & 6,55 & 7,07 & 0,19 & $100,0 \%$ \\
\hline $\mathbf{2 0 1 7}$ & 6,76 & 7,17 & 0,08 & $100,0 \%$ & 6,5 & 7,05 & 0,26 & $100,0 \%$ \\
\hline
\end{tabular}

Datos contemplados de cuatro (4) estaciones. S: desviación estándar.

Fuente: elaboración propia.

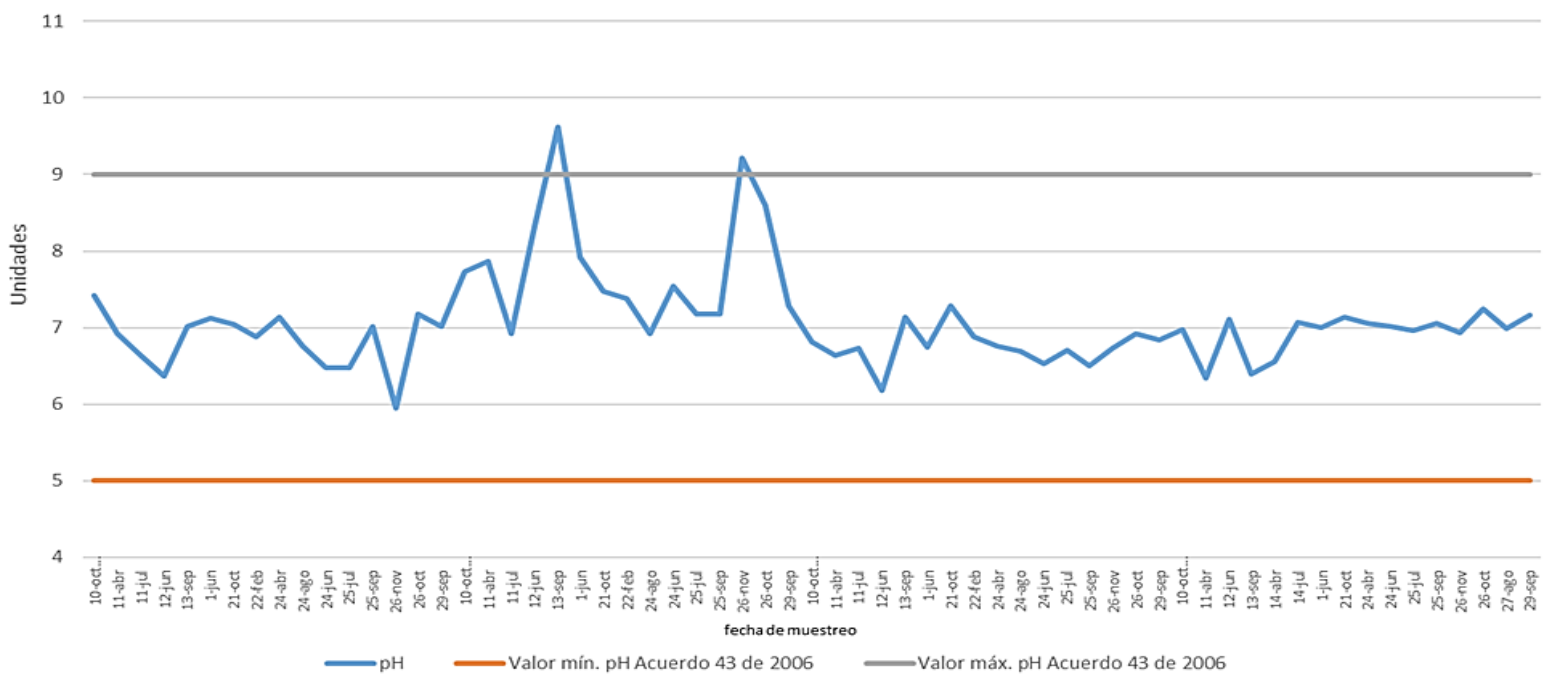

Figura 8. Comportamiento pH desde 2010 al 2017 vs. norma. Fuente: elaboración propia.

Finalmente, en la tabla 7, al compararse las concentraciones de sulfatos de los años estudiados con el límite máximo (400 mg/L) establecido en el Acuerdo 43/06, se determina baja influencia de este parámetro de interés sanitario en los puntos monitoreados en el río Bogotá.

Las concentraciones reportadas en el presente estudio pueden asociarse a su presencia natural en el agua [18] y también atribuirse a vertimientos domésticos, pues las aguas residuales domésticas presentan concentraciones entre 0,01 y $2,0 \mathrm{mg} / \mathrm{L}$, cantidades que en las actividades de riego pueden aportar a las características favorables a los suelos frente a los requerimientos nutricionales de los cultivos [20].

Vale la pena resaltar que de existir concentraciones más altas de sulfato, si se dan las condiciones limitadas de oxígeno y bajas concentraciones de nitratos como las que se analizaron anteriormente, el ion sulfato puede asociarse a problemas de olor a causa de su reducción bioquímica [18].

En la figura 9 se plasma el consolidado de los resultados para cada una de las mediciones contempladas de acuerdo con los datos analizados para los años 2007 al 2017.

\section{Conclusiones}

Los resultados de calidad de agua analizados en el presente estudio evidencian que las acciones desarrolladas por las autoridades ambientales y locales desde el año 2006 al 2019 no han sido suficientes para asegurar los objetivos de calidad en la cuenca alta del río Bogotá. 
Análisis multitemporal y estadístico de parámetros fisicoquímicos monitoreados en la cuenca alta del río Bogotá

Tabla 7. Concentraciones de sulfatos en $\mathrm{mg} \mathrm{SO}_{4}{ }^{-2} / \mathrm{L}$, cuenca alta desde 2010 al 2017

\begin{tabular}{|c|c|c|c|c|c|c|c|c|}
\hline Cuenca alta & \multicolumn{4}{|c|}{ Superior } & \multicolumn{4}{c|}{ Inferior } \\
\hline Año & Vr. Mín. & Vr. Máx. & Promedio & S & Vr. Mín. & Vr. Máx. & Promedio & S \\
\hline $\mathbf{2 0 1 0}$ & 3 & 3 & 3 & 0,00 & 3 & 19 & 7,9 & 6,52 \\
\hline $\mathbf{2 0 1 1}$ & 2 & 3 & 2,5 & 0,58 & 2 & 21 & 10,5 & 9,47 \\
\hline $\mathbf{2 0 1 2}$ & 2 & 19 & 6,95 & 8,14 & 2 & 22 & 12,93 & 9,25 \\
\hline $\mathbf{2 0 1 3}$ & 1 & 12 & 4,38 & 5,20 & 3,2 & 16 & 9,18 & 6,78 \\
\hline $\mathbf{2 0 1 4}$ & 0,58 & 2,8 & 1,69 & 1,57 & 5,5 & 13 & 9,25 & 5,30 \\
\hline $\mathbf{2 0 1 5}$ & - & - & - & - & - & - & - & - \\
\hline $\mathbf{2 0 1 6}$ & - & - & - & - & - & - & - & - \\
\hline $\mathbf{2 0 1 7}$ & 1,4 & 5,2 & 3,3 & 0,07 & - & 16 & N/A & N/A \\
\hline
\end{tabular}

S: desviación estándar; -: sin registro; N/A: no aplica. S: desviación estándar.

Fuente: elaboración propia.

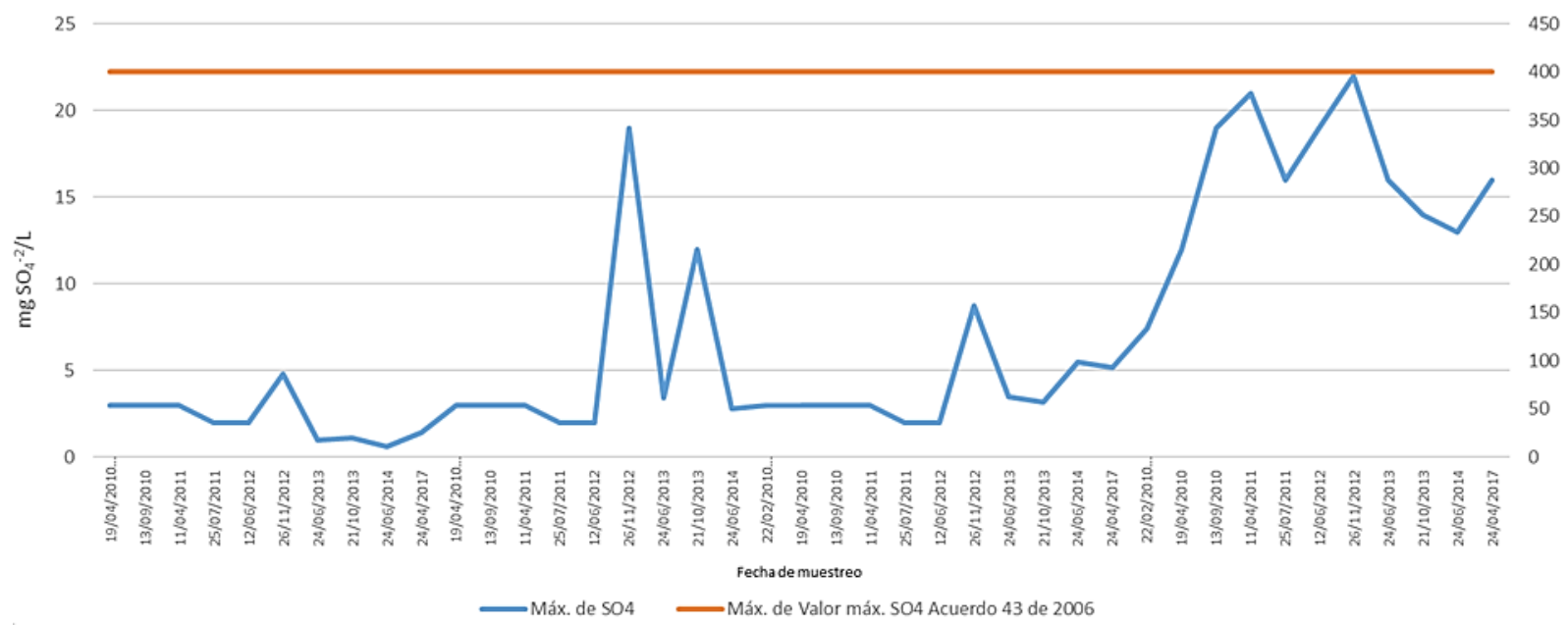

Figura 9. Comportamiento sulfatos desde 2010 al 2017 vs. norma. Fuente: elaboración propia.

Lo anterior demuestra la necesidad de fortalecer en la cuenca la gobernanza del agua en todas sus dimensiones, establecer estrategias articuladas con los diferentes usuarios de la cuenca en pro de una gestión integral del recurso hídrico, que permita reconocer su valor como resultado del fortalecimiento institucional para el seguimiento y control en pro de la protección y conservación del recurso, al igual que el fortalecimiento de la educación ambiental y el manejo de los conflictos entre los distintos actores de la cuenca.

Es recomendable construir plantas de tratamiento y que las existentes cumplan con los límites sugeridos en la normatividad ambiental; de igual manera, que los controles que ejercen las autoridades ambientales sobre los vertimientos sean en realidad estrictos para que de esta forma se dé cumplimiento total a los límites fijados en el Acuerdo 43/06.

El $92 \%$ de los datos de oxígeno disuelto en la cuenca alta superior excedió el límite mínimo establecido, mientras que en la parte inferior continúa siendo bajo al ser del
$30 \%$, lo que refleja un estado de degradación del río frente a este parámetro. Respecto al año 2006, las concentraciones menores de los años 2007 al 2017 estuvieron por debajo del dato mínimo, indicado en el Acuerdo 431/06.

El 87,9\% de los datos del compuesto $\mathrm{DBO}_{5}$ en la cuenca alta superior cumplió el límite máximo establecido, mientras que en la parte inferior descendió al 70,3\%. Respecto al año 2006, las concentraciones de los años 2007 al 2017 han disminuido con relación a lo indicado en el Acuerdo 431/06.

El porcentaje de cumplimiento de los sólidos suspendidos totales sigue siendo bajo en la cuenca alta superior del río Bogotá y casi nulo en la parte inferior, lo que refleja la afectación que continúa recibiendo la fuente hídrica por los vertimientos durante su recorrido, aún después de la expedición del Acuerdo 43 del 2006 de la CAR. 
El 90,6 \% de los datos de $\mathrm{pH}$ en la cuenca alta superior se encontró dentro del rango de calidad de 5-9 unidades, mientras que en la parte inferior se evidencia un cumplimiento del $100 \%$ de los datos analizados.

Los registros analizados de nutrientes, sulfatos y plomo permiten determinar que las concentraciones de estos parámetros en la cuenca alta del río Bogotá están acordes con los objetivos de calidad a cumplir en el año 2020.Una limitante del estudio realizado es que no fue posible la obtención de datos en todos los años del periodo analizado.

\section{Referencias}

[1] P. M. Vélez, "El reflejo del gigante en el agua: una historia ambiental del río Bogotá, 1950-2003”, Trabajo de grado, Universidad de los Andes, 2015.

[2] I. Barajas, J. Mahlknecht, J. Kaledin, M. Kjellén, A. Mejía, Aguas y ciudades en América Latina: Retos para el desarrollo sostenible. Inter-American Development Bank, 2018

[3] Corporación Autónoma Regional de Cundinamarca CAR, "Vive el Río Bogotá", S.F. [En Línea]. Disponible en:

https://www.car.gov.co/rio_bogota/vercontenido/5\#: :te $\mathrm{xt}=\mathrm{Los} \% 20$ vertimientos $\% 20$ industriales $\% 20 \mathrm{de} \% 201 \mathrm{as}, \mathrm{e}$ $1 \% 2080 \% 25 \% 20 \mathrm{de} \% 201 \mathrm{a} \% 20$ contaminaci\%C3\%B3n.

[4] Corporación Autónoma Regional de Cundinamarca CAR, "Evaluación Regional del Agua - ERA Cuenca Alta del río Bogotá", S.F. [En línea]. Disponible en: http://www.andi.com.co/Uploads/ERA\%20CUENCA\% 20ALTA\%20R\%C3\%8DO\%20BOGOT\%C3\%81.pdf.

[5] Secretaría Distrital de Planeación, “Aproximación a las implicaciones del Fallo del Consejo de Estado sobre el Río Bogotá”, 2014. [En línea]. Disponible en: http://www.sdp.gov.co/sites/default/files/aproximacion a_las_implicaciones_del_fallo_del_consejo_de_estado_ sobre_el_rio_bogota.pdf.

[6] A. Rodríguez, J. González, R. Suárez, "Bioacumulación por metales pesados en el capitán de la Sabana (Eremophilus mutisii), habitante de la cuenca alta del río Bogotá”, Revista electrónica de Ingeniería en Producción Acuícola, vol. 3, no. 3, 2007.

[7] A. Sanabria, C. Montenegro, M. Castro, D. Díaz, "Análisis multitemporal del índice de calidad del agua (WQI) en corrientes superficiales en la cuenca alta del río Bogotá”, Ingeniería Solidaria, vol. 13, no. 2, pp. 39-54, 2017, doi: 10.16925/in.v13i22.1751.
[8] Por el cual se establecen los objetivos de calidad del agua para la cuenca del río Bogotá a lograr en el año 2020, Acuerdo 43, 2006. [En línea]. Disponible en: https://www.alcaldiabogota.gov.co/sisjur/normas/Norm a1.jsp?i=22067.

[9] E. Chuvieco, Fundamentos de Teledetección Espacial, 2 ed. Madrid: Ediciones RIALP S.A, 1995.

[10] M. J. Jiménez-Moreno, M. J. González-Guillén, M. Escalona-Maurice, J. R. Valdez-Lazalde, C. A. AguirreSalado "Comparación de métodos espaciales para detectar cambios en el uso del suelo urbano", Revista Chapingo serie ciencias forestales y del ambiente, vol. 17, no. 3, pp. 389-406, 2011.

[11] Decreto Único Reglamentario del Sector Ambiente y Desarrollo Sostenible, Decreto 1076, 2015. [En línea]. Disponible en: www.parquesnacionales.gov.co/portal/wpcontent/uploads/2013/08/Decreto-Unico-ReglamentarioSector-Ambiental-1076-Mayo-2015.pdf.

[12] "Modelación dinámica de la calidad del agua del río Bogotá", EAAB-Universidad Nacional de Colombia, Informe Producto No. 2, 2009.

[13] J. Díaz, C. Granada, "Efecto de las actividades antrópicas sobre las características fisicoquímicas y microbiológicas del río Bogotá a lo largo del municipio de Villapinzón", Revista de la facultad de medicina, vol. 66, no. 1, pp. 45-52, 2016, doi: 10.15446/revfacmed.v66n1.59728.

[14] Gobernación de Cundinamarca - Secretaría de Ambiente y Universidad de La Salle, "Estrategias de producción más limpia en la cuenca alta del Río Bogotá", 2016. [En línea]. Disponible en: www.orarbo.gov.co/es/el-observatorio-y-losmunicipios/estrategias-de-produccion-mas-limpia-en-lacuenca-alta-del-rio-bogota.

[15] D. Aguirre, "Actualización de los Escenarios de Saneamiento del río Bogotá", trabajo de grado, Universidad de los Andes, Bogotá, 2015

[16] Corporación Autónoma Regional de Cundinamarca - CAR, "Río Bogotá: Adecuación hidráulica y recuperación ambiental", Evaluación Ambiental y Plan de Gestión Ambiental, vol. 1, no. 11-30, pp. 1-157, 2020.

[17] A. F. Suárez, C. A. García, M. L. Vaca, "Identificación y evaluación de la contaminación del agua por curtiembres en el municipio de Villapinzón", Tecnura, vol. 16, pp. 185-193, 2012. 
Análisis multitemporal y estadístico de parámetros fisicoquímicos monitoreados en la cuenca alta del río Bogotá

[18] C. Sierra, Calidad del agua: evaluación y diagnóstico. Medellín: Editorial de la Universidad de Medellín, 2011.

[19] C. N. Sawyer, P. L. McCarty, y G.F. Parkin, Química para la ingeniería ambiental, 4 ed. Bogotá: McGraw-Hill, 2000.

[20] E. González-Pérez, H. M. Ortega-Escobar, M. J. Yáñez-Morales, A. Rodríguez-Guillen, "Diagnóstico de indicadores de calidad fisicoquímica del agua en afluentes del río Atoyac", Tecnología y Ciencias Del Agua, vol. 10, no. 1, pp. 1-28, 2019, doi: $310.24850 / j-$ tyca-2019-01-02 